\title{
HNA-3a and HNA-3b antigens among 9 ethnic populations and the Han population in Southwest China
}

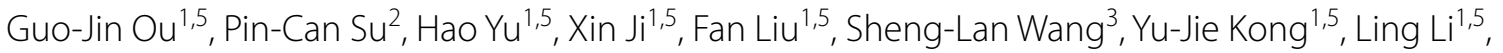
Jue Wang ${ }^{1,5^{*}}$, Zhong Liu ${ }^{1,5^{*}}$ and Willy Albert Flegel ${ }^{4}$

\begin{abstract}
Background: Human neutrophil antigen 3 (HNA-3) is encoded by the SLC44A2 gene. Antibodies against HNAs can cause severe, often fatal, transfusion reactions, known as transfusion-related acute lung injury, and neonatal neutropenia. We explored the 2 common HNA-3 variants in 9 ethnic populations residing in Sichuan and Yunnan provinces of China as compared to the Han population.

Methods: We genotyped for SLC44A2 (rs2288904) by polymerase chain reaction sequence-based typing among blood donors, for a total of 2206 individuals in Yunnan and 376 in Sichuan.

Results: The SLC44A2*02 allele (HNA-3b antigen) frequency varied between 0.24 and 0.33 for all 9 ethnic populations in Yunnan, including Zhuang, Derung, Hani, Lisu, Bai, Miao, Dai, Naxi, and Yi. Specifically, the Yi ethnicity did not present an unusually great $S L C 44 A 2^{*} 02$ frequency at any of the 4 locations examined in Yunnan. Except of the Yi ethnicity in Sichuan (0.40), the Han ethnicity, as the majority population group, had the greatest SLC44A2*02 frequency with 0.39 in Yunnan and 0.35 in Sichuan.

Conclusion: The ethnic populations in Southwest China are not at an increased risk for anti-HNA3a compared to the Han population, with the possible exception of Yi in Sichuan. Our data, however, corroborated the known high prevalence of SLC44A2*02 in Han populations. Hence, the Han populations in Yunnan, Sichuan and elsewhere in China are at a comparatively great risk for developing HNA-3a antibodies.
\end{abstract}

Keywords: HNA-3 antigens, TRALI, Gene polymorphism, PCR-SBT genotyping

\section{Background}

The human neutrophil antigen (HNA) system was established in 1998 by an International Society of Blood Transfusion (ISBT) Working Party [1, 2]. Nine HNAs have been identified, i.e., HNA-1a, $-1 b,-1 c,-3 a,-3 b,-4 a,-4 b,-5 a$, and $-5 \mathrm{~b}$. HNA-1, -4 , and -5 are expressed on glycoproteins CD16b (FcgR3b), CTL2, CD11b/18 (Mac-1, CR3, $\alpha \mathrm{M} \beta 2$-integrin), and CD11a (LFA-1, $\alpha \mathrm{M} \beta 2$-integrin), respectively [3]. Most HNA antigens are caused by

\footnotetext{
*Correspondence: 450071835@qq.com; liuz@ibt.pumc.edu.cn ${ }^{1}$ Clinical Blood Transfusion Research Center, Institute of Blood Transfusion, CAMS \& PUMC, No. 26 Hua-Cai Road, Chenghua District, Chengdu 610052, Sichuan, China

Full list of author information is available at the end of the article
}

single-nucleotide polymorphisms (SNPs) in the underlying genes, determining the different allelic forms.

HNA-3a and HNA-3b were identified in 1964 using antibodies from multiparous women that agglutinated leukocytes [4]. In 2010, the $S L C 44 A 2$ gene has been identified to express the HNA-3 antigens occurring on the choline transporter-like protein 2 (CTL2) [5]. The SLC44A2 gene has the rs2288904 single-nucleotide polymorphism, encoding the common HNA-3 protein variants Arg154 (HNA-3a) and Gln154 (HNA-3b), whichis used for SLC44A2 (rs2288904) genotyping.

HNA-3 is particularly important for the pathophysiology of transfusion-related acute lung injury (TRALI) and neonatal alloimmune neutropenia [6-8]. Individuals who lack the HNA-3a or HNA-3b antigens can be immunized 
and produce antibodies when exposed to the cognate antigen via blood transfusion or pregnancy. The HNA-3a antigen is particularly important owing to the association between anti-HNA-3a and TRALI [9].

Only a few cases of TRALI have been reported in China [10-12]. In a retrospective study among pediatric surgical patients, Xing et al. identified TRALI in two of 1495 transfusion cases [10]. Hence, the incidence of TRALI was higher than reported previously, which ranged from $1: 202,673$ in plasma to $1: 2,527,437$ in red blood cells [13]. Blood components from female donors are still used by hospitals in China. Anti-HLA were found among 5.6\% of nulliparous women [14], while $26.6 \%$ of female blood donors in China have a history of pregnancies. Despite the large numbers of plasma and platelet components transfused, the lack of awareness and diagnostic standards for TRALI, may lead to the failure of recognizing TRALI in China.

HNA-3 antigen frequencies vary among populations and countries. Population studies have shown that $13-19 \%[15,16]$ of Chinese Han individuals are negative for the HNA-3a antigen and are at risk for alloimmunization and development of anti-HNA-3a. There are 56 ethnic populations recognized in China. The Yi population of Xichang in the south of Sichuan province had the greatest frequency of blood donors at risk of harboring anti-HNA-3a [15] ever reported. To further explore the populations in Southwest China, we determined the frequencies of the alleles encoding HNA-3a (SLC44A2*01) and HNA-3b antigens (SLC44A2*02) in 9 ethnic populations residing in Sichuan and Yunnan provinces of China as compared to the Han population.

\section{Methods}

\section{Blood samples}

A total of 2582 samples were included in this study. Among them, 232 blood samples were obtained from Chinese Sichuan Han individuals collected at the Sichuan branch of the Chinese Marrow Donor Program (CMDP), and 144 Chinese Sichuan Xichang Yi blood donors collected at the Liangshan blood center. The Xichang Yi samples included the 100 samples previously reported samples by Chen et al. [15]. Another 2206 Yunnan individual samples including 196 Zhuang, 184 Derung, 190 Hani, 254 Lisu, 190 Bai, 209 Miao, 126 Dai, 187 Naxi, 83 Shilin Yi, 122 Luquan Yi, 120 Honghe Yi, 153 Chuxiong Yi and 192 Yunnan Han populations were collected at the Kunming blood center in Yunnan province (Fig. 1). The ethnicity were self-declared by the blood donors.

To ensure the correct ethnic background, we collected all blood samples in the local ethnic autonomous counties where the respective ethnic blood donors represented the vast majority of the population. Also, the volunteers were enrolled only after we carefully checked their identity cards documenting their residency in the county. Finally, blood donors were asked by our staff for their parents' ethnicity; unless both parents belonged to the local ethnicity, the blood donors were excluded from the study. All samples were collected after obtaining informed consent and the study was approved by the Ethics Committee of the Institution of Blood Transfusion (IBT), CAMS \& PUMC, and Kunming blood center in Yunnan province.

\section{Genotyping}

Genomic DNA was extracted from venous blood samples (TIANamp Blood DNA Kit; Tiangen, Beijing, China) at $20-50 \mathrm{ng} / \mu \mathrm{L}$ concentration. The SLC44A2 genotype (rs2288904) was examined by polymerase chain reaction (PCR)-sequence-based typing. The primers used to amplify a fragment of 291 bp containing the rs2288904 SNP from genomic DNA were as follows: forward, 5'-GGGCAGTGGCAGTGTACTA-3', and reverse, 5'-CATGCCCATCCTCATAGGTCG-3'. The PCR mix contained $1 \mu \mathrm{L}$ of DNA and $5 \mu \mathrm{L}$ of master mix (GoTaq Green; Promega, Madison, WI, USA) plus $5 \mu \mathrm{M}$ primers for a total volume of $10 \mu \mathrm{L}$. Thermal cycling conditions were as follows: $96{ }^{\circ} \mathrm{C}$ for $5 \mathrm{~min}$; 35 cycles at $96{ }^{\circ} \mathrm{C}$ for $30 \mathrm{~s}, 60^{\circ} \mathrm{C}$ for $30 \mathrm{~s}, 72^{\circ} \mathrm{C}$ for $45 \mathrm{~s}$; and a final extension at $72{ }^{\circ} \mathrm{C}$ for $7 \mathrm{~min}$. The PCR products $(3 \mu \mathrm{L})$ were resolved on $2 \%$ agarose gels and visualized under UV with ethidium bromide, followed by purification of PCR products $(7 \mu \mathrm{L})$ using Exo I and FastAP enzymes (Thermo; Vilnius, Lithuania). Sequencing was conducted with $1 \mu \mathrm{L}$ of purified PCR products (BigDye Terminator Ready Reaction v3.1 kit; Applied Biosystems, Foster City, CA, USA) using the reverse primer (ABI 3730 DNA Sequencer; Applied Biosystems). Standard thermal cycling conditions for sequencing reactions were used: $96{ }^{\circ} \mathrm{C}$ for $1 \mathrm{~min} ; 25$ cycles at $96^{\circ} \mathrm{C}$ for $10 \mathrm{~s}, 50^{\circ} \mathrm{C}$ for $5 \mathrm{~s}$, and $60^{\circ} \mathrm{C}$ for $4 \mathrm{~min}$.

\section{Statistical analysis}

$S L C 44 A 2 * 01$ and $S L C 44 A 2 * 02$ alleles observed in the 3 genotypes were counted and the allele frequencies calculated (Table 1). The Chi square test was used to exclude the possibility of deviations from the Hardy-Weinberg equilibrium in all 15 populations tested. Comparisons of allele frequencies between populations were performed using the Chi square test or Fisher's exact test (when the minimum expected frequency was less than five).

The chance of being exposed to HNA-3a by non-leukocyte-reduced blood components, while lacking this antigen, was calculated as $(2 \times S L C 44 A 2 * 01 \times S L C 44 A 2 *$ $\left.02+S L C 44 A 2 * 01^{2}\right) \times S L C 44 A 2 * 02^{2}$; the chance of being exposed to HNA-3b while lacking that antigen was calculated similarly. $P<0.05$ was considered significant. 


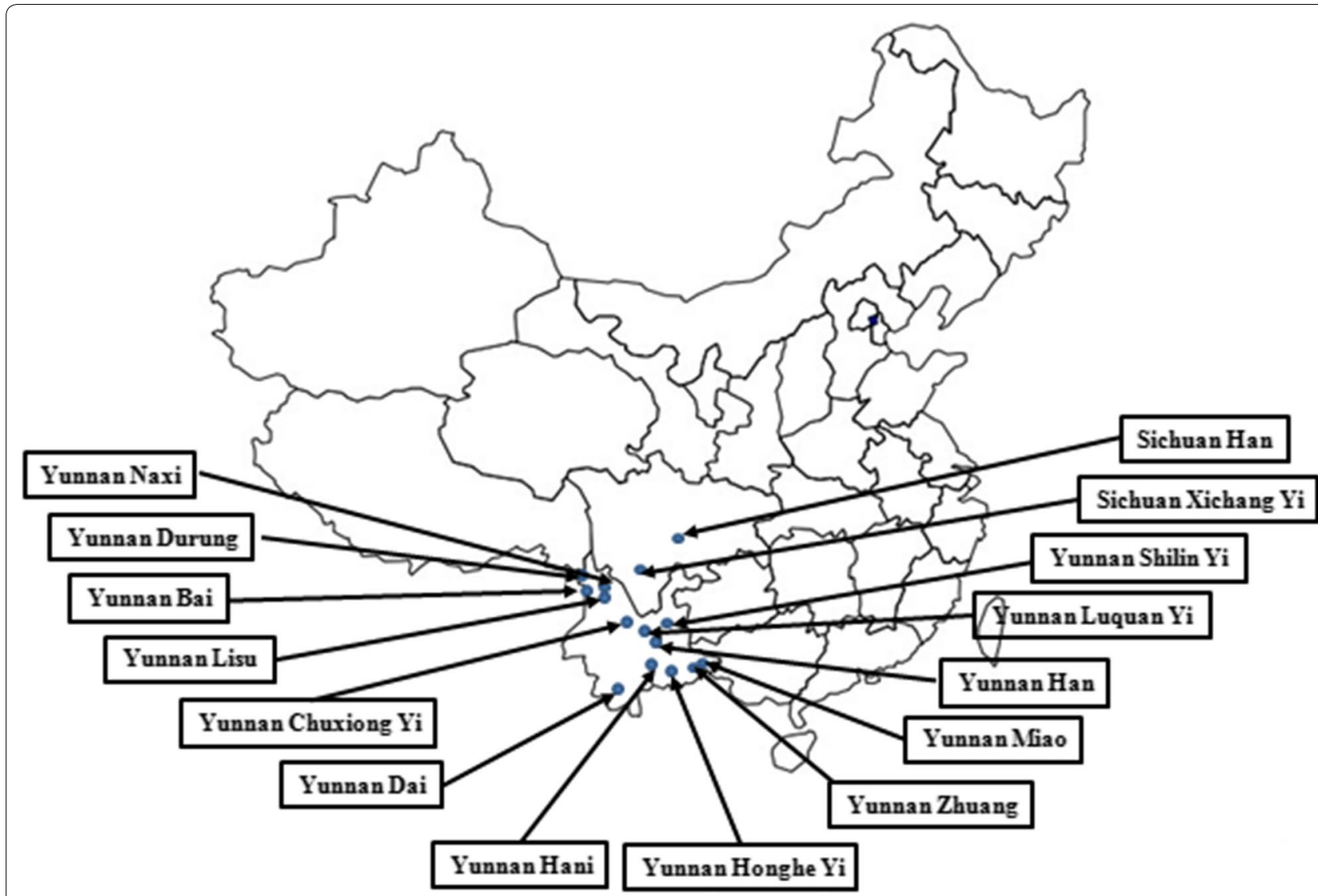

Fig. 1 The locations of the populations in Southwest China. Blood samples came from 9 ethnicities of the minority population groups in Yunnan province. Additional blood samples from the Yi ethnicity in Sichuan and the majority population group of Han ethnicity in Yunnan and Sichuan were collected for comparison

\section{Results}

We determined the frequencies of the 2 main $S L C 44 A 2$ alleles, encoding the 2 HNA-3 antigens, in 9 ethnic populations residing in the Sichuan and Yunnan provinces of China (Fig. 1). For comparison, the majority population group of Han ethnicity in Yunnan and Sichuan were tested, along with an enlarged sample for the Yi ethnicity in Sichuan.

\section{Ethnic populations in Southwest China}

The Yi ethnicity at any of the 4 locations in the Yunnan province did not present with an unusual great frequency of the SLC44A2*02 (HNA-3b) allele (Table 1). The 8 other ethnic populations in Yunnan, including Zhuang, Derung, Hani, Lisu, Bai, Miao, Dai, and Naxi, varied in SLC44A2*02 (HNA-3b) allele frequencies between 0.24 and 0.32 similar to the variability between 0.27 and 0.33 observed for Yi in Yunnan.

\section{Han population in Southwest China}

The Han population of Yunnan had the greatest frequency of the SLC44A2*02 (HNA-3b) allele with 0.39 (Table 1), barely short of Yi in Sichuan with 0.40 in this study and 0.41 as reported previously [15].

\section{Rate of exposure to the HNA-3a antigen}

The data allowed to calculate the chance of being exposed to the HNA-3a or HNA-3b antigen, while lacking the cognate antigen; each transfusion may pose an alloimmunization risk when non-leukocyte-reduced blood components are use (Table 1). These rates differed substantially but would strictly apply for transfusions among members of a given ethnicity, such as in directed transfusions, or to transfusions among Han majority population. Of note, the rate was consistently greatest for the Han population (Table 1), in accordance with all previously published SLC44A2 genotype data [15]. 


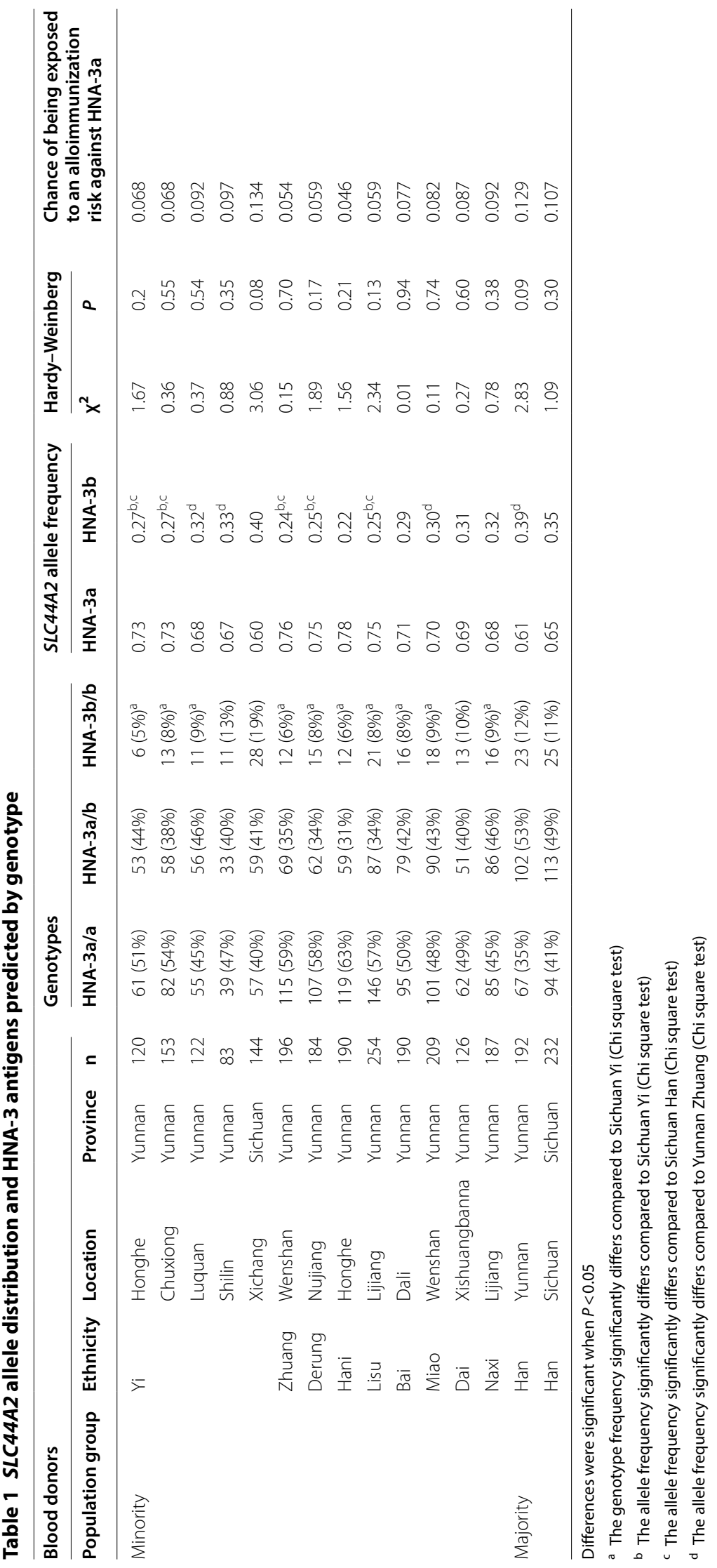




\section{Discussion}

The Yi population in the south of the Sichuan province had the greatest frequency of blood donors at risk of harboring anti-HNA-3a reported so far [15]. This finding prompted us to explore the alleles encoding HNA-3a (SLC44A2*01) and HNA-3b antigens (SLC44A2*02) in 9 ethnic populations residing in the immediately adjacent Yunnan province (Fig. 1). The SLC44A2*02 allele frequency varied between 0.24 and 0.33 for all 9 ethnic populations in Yunnan. Specifically, the Yi ethnicity did not present an unusually great SLC44A2*02 frequency at any of the 4 locations examined in Yunnan. Assuming the previously reported [15] unusual great frequency of the SLC44A2*02 among Yi in Sichuan represented a random fluctuation, we conclude that the 9 ethnic populations in Southwest China are not at an increased high risk for anti-HNA3a compared to the Han population.

The SLC44A2*02 frequencies in all populations examined in this study were significantly higher than in European [16-19], other Caucasian [20, 21], and American populations [22]. Our data corroborated the known high prevalence of HNA-3b in Han populations [23, 24]. Hence, the Han populations in Yunnan, Sichuan and elsewhere in China are at a higher risk for developing HNA3a antibodies compared to most populations outside of China [16-23]. We confirmed that the HNA-3b frequencies of Yi and Han residing close to each other in Sichuan were almost identical. Intermarriage among Yi and Han may have contributed and could have been more prevalent in Sichuan compared to the adjacent Yunnan province. The number of individuals per ethnic population ranged, with 1 exception, from 120 to 254 (Table 1 ) and should be large enough to allow an approximation. Particularly, the SLC44A2 (HNA-3a and HNA-3b) alleles were in Hardy-Weinberg equilibrium in all 15 locations tested representing 10 distinct ethnic populations, making stochastic sampling errors unlikely.

The prevalence of individuals in China, who are at risk of developing anti-HNA-3a, may be considered for clinical decisions, such as excluding plasma donations by previously pregnant females, because TRALI is currently the most common cause of transfusion-related deaths in Western health care systems, and approximately $80 \%$ of reported TRALI cases are associated with the transfusion of blood products containing unsuspected leukocytespecific alloantibodies [15], mainly anti-HNA or antiHLA. A retrospective study of 36 TRALI cases showed that HNA antibodies, especially anti-HNA-3a, accounted for 10 of 12 HNA antibody-mediated reactions and 6 of 10 fatalities, including one that occurred after the transfusion of red blood cells. Antibodies with specificity against HNA-1a and HNA-2a were only detected in 2 of 36 TRALI cases [7]. HNA-3a alloantibodies are prone to cause severe and often fatal clinically relevant complications [25-27]. Individuals expressing only the HNA-3b antigen can form antibodies against the HNA-3a antigen, whereas individuals expressing only the HNA-3a antigen can form antibodies against the HNA-3b antigen.

Only the Japanese population (37\% for HNA-3b) [28], has been reported to have HNA-3b frequencies similar to Chinese populations. Other Asian populations, such as Thai (31\%) [29, 30] and Asian Indian (24\%) [22], resemble the Caucasian prevalences, which interestingly was also reported in a large cohort of 493 Han individuals in Guangzhou (26\%) [24]. Anti-HNA-3a were found in $0.23 \%$ of previously pregnant women, reflecting an immunization rate of $7 \%$, in the population of Germany where $4 \%$ can be expected to be HNA-3a negative.

Compared to any Western population [16-21], the chance of being exposed to an alloimmunization risk against HNA-3a is 2 times greater in ethnic populations, but 2-3 times greater in the Han population. Assuming a $0.23 \%$ prevalence similar to Western studies, up to $1,000,000$ women in China may be at risk of HNA-3a alloimmunization. Among 14,000,000 blood donors in 2016 female donors comprised 30\%, of whom 10,000 may harbor anti-HNA-3a $(14$ mio $\times 30 \% \times 0.23 \%)$. However, anti-HNA-3a causing TRALI has been rarely reported in China [10]. If anti-HNA-3a and TRALI in China were truly less common than in Western settings, this should prompt research for the biologic reason, such as possible protective HLA haplotypes prevalent in China but lacking in Western populations; this research may also address the unexplained observation why the immunization rate for anti-HNA-3b $(0.7 \%)$ is so much smaller than for anti-HNA-3a (7\%) [31]. If anti-HNA-3a and TRALI are more common, as they may be in China, improved hemovigilance in combination with prevention strategies should be evaluated and implemented similar to Western health care systems. Also, detection of HLA or HNA antibodies in blood donors, especially in women with childbearing history, can definitely improve blood transfusion safety in China.

\section{Conclusion}

More studies in Chinese populations may be worthwhile to evaluate the risk and possible need to prevent the occurrence of TRALI. The risk of TRALI caused by HNA-3a antibodies can be reduced by excluding female blood donors from plasma and platelet donations or any donor who is homozygous for the $S L C 44 A 2 * 02$ allele in China [15]. 


\section{Abbreviations}

TRALI: transfusion-related acute lung injury; HNA: human neutrophil antigen; CMDP: Chinese Marrow Donor Program; ISBT: International Society of Blood Transfusion.

\section{Authors' contributions}

OGJ, YH, LF, JX, KYJ: performed the experiments; WJ: experimental design and quality control of experiments; WSL, SPC: collection and determination of samples; OGJ, LZ: wrote the original manuscript draft; LZ: experimental design, data analysis and discussion; FWA: data analysis, discussion of the results and substantial revision of the manuscript. All authors read and approved the final manuscript.

\section{Author details}

${ }^{1}$ Clinical Blood Transfusion Research Center, Institute of Blood Transfusion, CAMS \& PUMC, No. 26 Hua-Cai Road, Chenghua District, Chengdu 610052, Sichuan, China. ${ }^{2}$ Transfusion Medicine Research Department, Yunnan Kunming Blood Center, Kunming, China. ${ }^{3}$ Blood Center of the Liangshan Autonomous Region, Sichuan, China. ${ }^{4}$ Department of Transfusion Medicine, $\mathrm{NIH}$ Clinical Center, National Institutes of Health, Bethesda, MD, USA. ${ }^{5}$ Key Laboratory of Transfusion Adverse Reactions, CAMS, Chengdu, Sichuan, China.

\section{Acknowledgements}

The authors appreciate the study participants for generously providing venous blood samples used in this study. We thank Kshitij Srivastava for critical review of the manuscript.

\section{Competing interests}

The authors declare that they have no competing interests.

\section{Consent for publication}

Not applicable.

\section{Data availability}

All the data and materials supporting the conclusions were included in the main paper.

\section{Disclaimers}

The recommendations and opinions expressed are those of the authors, not their institutions or organizations. The views expressed do not necessarily represent the view of the National Institutes of Health, the Department of Health and Human Services, or the US Federal Government.

\section{Ethics approval and consent to participate}

This study was approved by the ethic committees of the Institution of Blood Transfusion (CAMS \& PUMC) and Kunming blood center which was conducted according to the principles of the Declaration of Helsinki. All participants provided written informed consent before enrolment, and the study's protocol was approved by the ethic committees of the Institution of Blood Transfusion (CAMS \& PUMC)

\section{Funding}

This work was supported by the Funding of Sichuan Science and Technology under Contract 2017RZ0047 and the CAMS Innovation Fund for Medical Sciences under Contract 2016-12M-3-024 provided us some blood samples and HNA-3 genotyping technology, funding of Kunming Science and Technology Bureau project, Grant Number: 20130402AS012141I and Ministry of Science and Technology of China, Grant/Award Number: 2014EG150133 provide us blood samples from minor populations. This work was supported by the Intramural Research Program (Project ID Z99 CL999999) of the NIH Clinical Center.

\section{Publisher's Note}

Springer Nature remains neutral with regard to jurisdictional claims in published maps and institutional affiliations.

Received: 15 January 2018 Accepted: 7 March 2018

Published online: 14 March 2018

\section{References}

1. Bux J. Nomenclature of granulocyte alloantigens. ISBT working party on platelet and granulocyte serology, granulocyte antigen working party. International Society of Blood Transfusion. Transfusion. 1999;39:662-3.

2. Bux J. Human neutrophil alloantigens. Vox Sang. 2008;94:277-85.

3. Moritz E, Norcia AM, Cardone JD, Kuwano ST, Chiba AK, Yamamoto M, Bordin JO. Human neutrophil alloantigens systems. Anais da Academia Brasileira de Ciências. 2009;81:559-69.

4. Van Leeuwen A, Eernisse JG, Van Rood JJ. A new leucocyte group with two alleles: leucocyte group five. Vox Sang. 1964;9:431-46.

5. Greinacher A, Wesche J, Hammer E, Furll B, Volker U, Reil A, Bux J. Characterization of the human neutrophil alloantigen-3a. Nat Med. 2010;16:45-8.

6. Curtis BR, Cox NJ, Sullivan MJ, Konkashbaev A, Bowens K, Hansen K, Aster $\mathrm{RH}$. The neutrophil alloantigen HNA-3a (5b) is located on choline transporter-like protein 2 and appears to be encoded by an R>Q154 amino acid substitution. Blood. 2010;115:2073-6.

7. Kopko PM, Marshall CS, MacKenzie MR, Holland PV, Popovsky MA. Transfusion-related acute lung injury: report of a clinical look-back investigation. JAMA. 2002:287:1968-71.

8. Reil A, Keller-Stanislawski B, Gunay S, Bux J. Specificities of leucocyte alloantibodies in transfusion-related acute lung injury and results of leucocyte antibody screening of blood donors. Vox Sang. 2008;95:313-7.

9. Davoren A, Curtis BR, Shulman IA, Mohrbacher AF, Bux J, Kwiatkowska BJ, McFarland JG, Aster RH. TRALI due to granulocyte-agglutinating human neutrophil antigen-3a (5b) alloantibodies in donor plasma: a report of 2 fatalities. Transfusion. 2003;43:641-5.

10. Xing Z, Wang QS, Yang QN, Meng LX, Tong HX. Transfusion-related acute lung injury in paediatric surgical patients: a retrospective study. Transfus Apher Sci. 2014;51(2):215-8.

11. Wang QS, Yang QN, Lin Q. Research and analysis transfusion related respiratory adverse reactions in adult surgery patients. Chin J Blood Transfus. 2016;29(12):1345-9 (Chinese journal).

12. An QX, An N, Chen XP. Analysis of 24 cases with transfusion-related acute lung injury in chinese literatures. Chin J Blood Transfus. 2013;15(3):228-32 (Chinese journal)

13. Eder AF, Herron R, Strupp A, et al. Transfusion-related acute lung injury surveillance (2003-2005) and the potential impact of the selective use of plasma from male donors in the American Red Cross. Transfusion. 2007:47(4):599-607.

14. Xu XZ, Xia WJ, Chen DW, et al. Detection and analysis of HLA antibodies and HNA-3a antibodies in blood donors in Guangzhou area. Chin J Blood Transfus [China]. 2015;28(11):1337-9.

15. Chen Q, Srivastava K, Liu Z, Xiao J, Huang C, Sun J, Li M, Flegel WA. Genotype frequency of human neutrophil antigen-3 polymorphisms in the Yi, Han, and Tibetan populations of China. Transfusion. 2016;56:737-42.

16. Lopes LB, Baleotti W Jr, Suzuki RB, Fabron A Jr, Chiba AK, Vieira-Filho JP, de Souza Castro B, Midori Kunioshi A, Bordin JO. HNA-3 gene frequencies in Brazilians and a new polymerase chain reaction-restriction fragment length polymorphism method for HNA-3a/3b genotyping. Transfusion. 2014:54:1619-21.

17. Norcia AM, Sugano EY, Chiba AK, Moritz E, Guirao FP, Yamamoto M, Bordin JO. Human neutrophil alloantigen-1a, $-1 \mathrm{~b},-2,-3 \mathrm{a}$ and $-4 \mathrm{a}$ frequencies in Brazilians. Tissue Antigens. 2009;74:404-7.

18. Nielsen KR, Koelbaek MD, Varming K, Baech J, Steffensen R. Frequencies of HNA-1, HNA-3, HNA-4, and HNA-5 in the Danish and Zambian populations determined using a novel TaqMan real time polymerase chain reaction method. Tissue Antigens. 2012;80:249-53.

19. Hauck B, Philipp A, Eckstein R, Ott S, Zimmermann R, Dengler T, Zingsem J. Human neutrophil alloantigen genotype frequencies among blood donors with Turkish and German descent. Tissue Antigens. 2011:78:416-20.

20. Cardoso SP, Chong W, Lucas G, Green A, Navarrete C. Determination of human neutrophil antigen-1,-3,-4 and -5 allele frequencies in English Caucasoid blood donors using a multiplex fluorescent DNA-based assay. Vox Sang. 2013;105:65-72.

21. Bowens KL, Sullivan MJ, Curtis BR. Determination of neutrophil antigen HNA-3a and HNA-3b genotype frequencies in six racial groups by highthroughput 5'exonuclease assay. Transfusion. 2012;52:2368-74. 
22. Huvard MJ, Schmid P, Stroncek DF, Flegel WA. Frequencies of SLC44A2 alleles encoding human neutrophil antigen-3 variants in the African American population. Transfusion. 2012;52:1106-11.

23. He J, Zhang W, Wang W, Chen N, Han Z, He J, Zhu F, Lv H. Genotyping of human neutrophil antigens by polymerase chain reaction sequencebased typing. Blood Transfus. 2014;12(Suppl 1):s292-8.

24. Xia W, Bayat B, Sachs U, Chen Y, Shao Y, Xu X, Deng J, Ding H, Fu Y, Ye $X$, Santoso $S$. The frequencies of human neutrophil alloantigens in the Chinese Han population of Guangzhou. Transfusion. 2011:51:1271-7.

25. Middelburg RA, van Stein D, Briet E, van der Bom JG. The role of donor antibodies in the pathogenesis of transfusion-related acute lung injury: a systematic review. Transfusion. 2008;48:2167-76.

26. Seeger W, Schneider U, Kreusler B, von Witzleben E, Walmrath D, Grimminger F, Neppert J. Reproduction of transfusion-related acute lung injury in an ex vivo lung model. Blood. 1990;76:1438-44.
27. Muniz M, Sheldon S, Schuller RM, Young NS, Klein HG, Leitman SF, Stroncek DF. Patient-specific transfusion-related acute lung injury. Vox Sang. 2008;94:70-3.

28. Matsuhashi M, Tsuno NH, Kawabata M, Mishima Y, Okochi N, Santoso S, Tozuka M, Takahashi $K$. The frequencies of human neutrophil alloantigens among the Japanese population. Tissue Antigens. 2012;80:336-40.

29. Changsri K, Tobunluepop P, Songthammawat D, Apornsuwan T, Kaset C, Nathalang O. Human neutrophil alloantigen genotype frequencies in Thai blood donors. Blood Transfus. 2014;12(Suppl 1):s286-91.

30. Nathalang O, Intharanut K, Siriphanthong K, Nathalang S, Leetrakool N. Risk estimation of HNA-3 incompatibility and alloimmunization in Thai populations. PLoS ONE. 2015;10:e0116905.

31. Reil A, Wesche J, Greinacher A, Bux J. Geno- and phenotyping and immunogenicity of HNA-3. Transfusion. 2011;51(1):18-24.

\section{Submit your next manuscript to BioMed Central and we will help you at every step:}

- We accept pre-submission inquiries

- Our selector tool helps you to find the most relevant journal

- We provide round the clock customer support

- Convenient online submission

- Thorough peer review

- Inclusion in PubMed and all major indexing services

- Maximum visibility for your research

Submit your manuscript at www.biomedcentral.com/submit 